\title{
«Avec la meilleure fidélité de mon cœur»: lettere del «civis aretinus» Pierre de Nolhac a Vittorio
} Cian

\section{Clara Allasia}

\section{(2) OpenEdition Journals}

\section{Edizione digitale}

URL: http://journals.openedition.org/studifrancesi/7793

DOI: 10.4000/studifrancesi. 7793

ISSN: 2421-5856

\section{Editore}

Rosenberg \& Sellier

\section{Edizione cartacea}

Data di pubblicazione: 1 juillet 2009

Paginazione: 279-297

ISSN: 0039-2944

\section{Notizia bibliografica digitale}

Clara Allasia, ««Avec la meilleure fidélité de mon cœur»: lettere del «civis aretinus» Pierre de Nolhac a Vittorio Cian», Studi Francesi [Online], 158 (LIII | II) | 2009, online dal 30 novembre 2015, consultato il 13 janvier 2021. URL: http://journals.openedition.org/studifrancesi/7793 ; DOI: https://doi.org/10.4000/ studifrancesi.7793

\section{(c)}

Studi Francesi è distribuita con Licenza Creative Commons Attribuzione - Non commerciale - Non opere derivate 4.0 Internazionale. 


\section{"Avec la meilleure fidélité de mon cour»: lettere del «civis aretinus» Pierre de Nolhac a Vittorio Cian}

Il $1^{\circ}$ ottobre 1931 comparve sulla «Revue de Paris» un «conte philosophique», così lo definiva il suo autore, destinato a suscitare violentissime polemiche nell'Italia fascista. La nuit de Pie XII si apre su una «nuit d'été romane sèche et brûlante», improvvisamente sconvolta dalla notizia che «le Duce venait d'être assassiné». Pio XII vede dal suo studio «trois gerbes de flamme jaillir sur la cité [...]: le ministère de l'Intérieur, le Central des communications, et sans doute le Palais de Venise». Ben conoscendo «la terreur qui s'abattit sur Rome, le jour de la mort de César», il pontefice viene convinto a fuggire dal timore che «la révolution» possa divenire «maîtresse de Rome». Arrivato in incognito ad Avignone chiede ospitalità al maire che appartiene a un «parti socialiste-révolutionnaire, le plus modéré d'Avignon», ed è abitualmente «courtois pour les étrangers». Pur non riconoscendo gli illustri ospiti e pensando si tratti di «deux vieux fous», il maire li indirizza al « $\mathrm{D}^{\mathrm{r}}$ Colombe», «l'historien savant du monument» che sovrintende al palazzo papale.

Nonostante la vicenda si riveli un sogno fatto dal pontefice «dans son grand cabinet de travail» e si concluda con la notizia che «le Duce, en parfaite santé, venait de monter à cheval pour sa promenade quotidienne», il conte scatenò in Italia un'ondata di violenta ostilità nei confronti di Nolhac, appena insignito della cittadinanza onoraria di Arezzo e del Grande Ufficialato della Corona d'Italia.

«Il est hardi, mais c'est du côté de "L'Osservatore Romano", que j'attendrai les foudres, et non pas du "Tevere" et de ses amis...»" scriveva stupito Nolhac, nei giorni in cui sceglieva di rispondere dalle pagine della «Tribuna», dichiarando di aver voluto «scrivere un racconto sul genere di quelli di Alfonso Daudet», per dipingere «il popolino di Avignone, entusiasta, credulo, acceso, [che] spera da cinque secoli nel ritorno di un Papa tra le sue mura». E aggiungeva di conoscere «certi letterati provenzali», che «si augurerebbero di veder rinnovarsi l'augusta visita nel palazzo di Clemente VI».

L'autore, che dichiarava di avere sul suo tavolo da lavoro «due ritratti [...] quelli di Dante e del Duce: le due grandi figure latine» ${ }^{2}$, confessava a Vittorio Cian di com-

(1) Parigi, 7 ottobre 1931. Le due citazioni del titolo appartengono invece alle lettere da Parigi, 10 giugno 1933 e 2 novembre 1931. Tutte le lettere di Pierre de Nolhac (socio straniero della classe di Scienze morali dell'Accademia delle Scienze di Torino dal 23 giugno 1918) qui citate sono inedite; esse provengono dal Fondo Cian dell'Accademia delle Scienze di Torino (d'ora in poi denominato FCAS), che ne custodisce 134 (dal 1889 al 1935), più una della moglie Alix del 1894, mentre due, del 1918 e del 1935, sono presso il Fondo Cian della Fondazione Cini di Venezia (FCFC). Al momento risultano disperse le lettere in risposta a quelle inviate da Cian nel 1885 e trascritte nell'introduzione a Un'amicizia petrarchesca. Carteggio Nolbac-Novati, a cura di A. Brambilla, Padova, Antenore, 1988, pp. VII-XI. Di ogni lettera si segnalano il luogo e la data, inserite fra quadre se desunte dal timbro postale. Sono state rispettate le abbreviazioni e i modi di scrittura dell'originale salvo che nell'oscillazione virgolette/corsivi per indicare i titoli. Per le caratteristiche complessive del carteggio Cian mi permetto di rimandare a C. Allasia, Prime spigolature dal Fondo Cian dell'Accademia delle Scienze di Torino, «Memorie dell'Accademia delle Scienze di Torino», 29 (2005), pp. 3-58.

(2) Pierre de Nolhac e l'Italia fascista, «La Tribuna», 14 ottobre 1931 . 
prendere solo in parte l'ostilità suscitata e di aver pertanto particolarmente apprezzato la «lettre si franche et si loyale» inviatagli per l'occasione dal «vieux ami». Grazie alle di lui parole si era «mieux éclairé $[. .$.$] une question pénible», che «les articles de$ journaux» non avevano saputo illustrare, e Nolhac aveva toccato con mano «comme il est difficile de se comprendre entièrement de peuple à peuple!».

E aggiungeva:

Aurai-je jamais cru blesser, par la fantaisie innocente d'un vieux poète, des cœurs auxquels je suis si attaché. Cette histoire, qui n'est que l'enchaînement d'imaginations toutes invraisemblables, exigeait au début l'invraisemblance qui déclenchait, qui déterminait toutes les autres. C'est le système que je suis dans les «contes philosophiques» que j'écris en ce moment. Celui-ci m'a valu de violentes attaques antifascistes en ce moment avec la lettre qui a suivi. Certains français, d'autre part, y ont vu une thèse fasciste: la nécessité du maintien du régime pour la vie de l'Eglise.

Je n'ai rien soutenu du tout et quant à mon respect pour le Duce et à ma conviction profonde de la solidité des institutions, j'en donne assez de preuves, tous les jours, par écrit et de vive voix ${ }^{3}$.

Nonostante a questa data Cian mostrasse un'incrollabile fede nel regime e in Mussolini, aveva perfettamente individuato, a differenza di molti altri, i sentimenti ispiratori della Nuit. E ciò non stupisce, se si tiene conto che disponeva di tutti gli elementi necessari, sulla scorta di una conoscenza iniziata ben quarantasei anni prima, nel 1885 , a cui si associava il netto ricordo «d'un sentimento d'invidia [...] verso lo studioso straniero - borsista dell'École Française appena insediatasi a Palazzo Farnese - che aveva potuto [...] giungere al ritrovamento dell'autografo del Canzoniere Petrarchesco». Dal canto suo Cian, «sebbene impedito allora di tentare le desiderate ricerche nella Biblioteca Vaticana, aveva sostenuta vivamente» nella sua tesi di laurea «l'esistenza di quel manoscritto e l'uso fattone da Pietro Bembo» ${ }^{4}$. Il «jeune et aimable italien», ammette Nolhac nel suo Journal, «a compris l'intérêt du manuscrit que j'avais sottement regardé» ${ }^{5}$. Così, forse anche per pagare il debito da lui sempre ammesso con onestà, Nolhac aveva recensito con favore sulla «Revue critique» ${ }^{6}$ la tesi di laurea di Cian, «paradigmatica di ogni ulteriore ricerca sull'argomento» ${ }^{7}$. Quest'ultimo, a sua volta, aveva provveduto a disegnare, per il «Giornale», una mappa di parte dell'«arcipelago d'articoli» ${ }^{8}$, che il francese andava allestendo parallelamente alla ste-

(3) [Paris], 2 novembre 1931.

(4) V. Cian, Critica letteraria e cavalleria internazionale: lettera aperta a Pier de Nolhac, «Gazzetta piemontese sera», 14-15 aprile 1893. Per ulteriori particolari su questa singolare concorrenza di intuizioni, rimando ai contributi citati alla nota 11 .

(5) Journal de Pierre de Nolhac après son mariage, in G. Zucchelli, Pierre de Nolhac et l'Italie, Saigon, Saigon An-Quan, 1971, p. 246.

(6) La recensione comparve sulla «Revue critique d'histoire et de littérature», IV (1886), e venne ripresa dal «Giornale Storico», con un ampio stralcio: «On travaille beaucoup et bien en Italie depuis quelques années. L'étude de la littérature nationale est, plus que tout autre, à l'ordre du jour, et une excellente revue, $[\ldots]$ le "Giornale Storico", recueille les documents, éclaircit les questions, réunit en un mot les matériaux d'une grande et définitive histoire littéraire de l'Italie. Un des jeunes collaborateurs de cette revue vient de publier un intéressant travail, inspiré par le même esprit de critique sincère et d'enthousiasme éclairé, et qui apporte une contri- bution précieuse à la connaissance du XVI ${ }^{\mathrm{e}}$ siècle», VIII (1886), p. 476.

(7) C. Dionisotti, rec. a V. Cian, Un illustre nunzio pontificio del Rinascimento: Baldassar Castiglione, «Giornale storico della letteratura italiana», LXIX (1952), p. 33.

(8) G. Billanovich, Nolhac e Petrarca, in Studi di letteratura e di storia in memoria di Antonio Di Pietro, Milano, Vita e Pensiero, 1977, p. 322. Le recensioni di Cian comparse sul «Giornale storico» riguardano i Fac-similés de l'écriture de Pétrarque et Appendices au "Canzoniere" autographe, V (1887), pp. 441-448, Les correspondants d'Alde Manuce. Matériaux nouveaux d'bistoire littéraire (1483-1514), VII (1889), pp. 391-398. Più corposa quella su La bibliothèque de Fulvio Orsini, VI (1888), pp. 230-249, in cui Cian delega parzialmente alle note il compito di segnalare qualcuna delle «tante e tali imprecisioni che lo fanno elencare - a differenza del Pétrarque - tra i libri da usarsi con cautela», G. Billanovich, Nolhac e Petrarca, cit., p. 320. 
sura del suo capolavoro, Pétrarque et l'Humanisme 9 . Non c'è traccia di risentimento, neppure nella recensione del Pétrarque, pubblicata da Cian nel 1892 sulla «Rivista storica italiana», perché per il «Giornale Storico» spettava a Francesco Novati, allora condirettore, firmare il compte-rendu. Cian racconta, nel necrologio di Nolhac, che Novati rinunciò a pubblicare la sua recensione perché «accintosi con tanto impegno e tanta larghezza di preparazione» vi aveva «indugiato troppo a lungo, finendo col trovarsi di fronte ad un ritardo così grave, da parergli un ostacolo insormontabile» ${ }^{10}$. In realtà, lo ha già suggerito a suo tempo Alberto Brambilla ${ }^{11}$, le cose andarono diversamente. Novati, impegnato nella non facile edizione della Navigatio Sancti Brendani, dava l'impressione di non voler entrare più di tanto in una polemica che si era preannunciata fin dalla pubblicazione, nel 1886, dell'intervento ${ }^{12}$ di Nolhac all'“Académie des Inscriptions et des belles lettres”, in cui lo studioso affermava con sicurezza che il Vat. Lat. 3195, da lui individuato, era l'antigrafo dell'edizione aldina del 1501. Si trattava, com'è noto, di un errore, e uno scrittore della Biblioteca Vaticana, Giuseppe Salvo-Cozzo ${ }^{13}$, lo aveva messo in luce con modi che rasentavano la villania.

Cian provvide a «consoler pleinement» Nolhac «de cette petite amertume, bien petite, en vérité» ${ }^{14}$, dando vita a «une polémique de quatre numéros dans la Gazetta [sic] Piemontese» ${ }^{15}$ dal significativo titolo Critica letteraria e cavalleria internazionale: lettera aperta a Pierre de Nolhac ${ }^{16}$. In essa si esprimeva la solidarietà della scuola storica, irritata della brochure di Salvo-Cozzo, «pleine du fiel de certains bibliothécaires que je reconnais $\gg^{17}$. D'altronde l'autore aveva, per stessa ammissione di Nolhac, portato «la seule attaque qui demeure justifiée [...] de la collation faite sans conscience ...par Ernest Langlois» che gli aveva permesso di stabilire, erroneamente aggiunge Nolhac, «l'identité de l'Aldine et du manuscrit» ${ }^{18}$.

Je reçois la brochure Salvo-C. [...]. La thèse principale (l'imposture d'Alde et de Bembo) ne me semble pas du tout établie. C'est un plaidoyer d'avocat qui tronque et dénature les documents (la lettre de 1544 par ex.). Il est toutefois certain que j'ai été mal servi par Langlois dans des collations ou plutôt qu'il a jugé insignifiantes des variantes qui ne le sont pas. Je n'oserais juger définitivement sans avoir sous les yeux les mss., car plusieurs points essentiels (les variantes du 3197 par ex.) semblent laissées volontairement hors du débat par l'avocat sicilien. Le précieux 3195 n'était évidemment pas dans la poussière de l'atelier, entre les mains des protes, ils devaient avoir sous les yeux le 3197 avec les variantes qu'ils devaient suivre. Leur travail n'a pas été aussi consciencieux qu'Alde l'a cru ou laissé croire (et en ce temps là, on se satisfaisait à

(9) Silvia Fabrizio-Costa discorre dell'opportunità, suggeritale anni fa da Marziano Guglielminetti, di una traduzione italiana della seconda edizione del Pétrarque, in Parlando di Petrarca..., in Maître et passeur. Per Marziano Guglielminetti dagli amici di Francia, a cura di C. Sensi, pres. di L. SozZI, Alessandria, Ed. dell'Orso, 2008, pp. 41-55. Ringrazio Claudio Sensi per avermi segnalato il contributo quando il volume era ancora in bozze.

(10) V. Cian, Necrologi: Pierre de Nolhac, «Giornale storico della letteratura italiana», LIV (1936), p. 189.

(11) Sulla questione si veda la lettera di Novati a Cian pubblicata da Brambilla (Un'amicizia petrarchesca, cit., p. XXIII). Novati avrebbe poi recensito il Pétraque sulla «Perseveranza» del 30 gennaio 1893.

(12) P. DE NolHac, Le "Canzoniere" autographe de Pétrarque, Paris, C. Klincksieck, 1886; l'opuscolo era stato preceduto, il 4 gennaio 1886 , dall'annuncio della scoperta sulla «Revue critique». Su tutta la questione e sulla prudenza invece espres- sa dal D'Ancona e dal Monaci si cfr. G. FRAsso, Appunti sul Petrarca Aldino del 1501, in Vestigia: studi in onore di Giuseppe Billanovich, a cura di R. Avesani, M. Ferrari, T. Foffano, G. Frasso e A. SotTILI, Roma, Edizioni di storia e letteratura, 1984, pp. 315-317.

(13) Giuseppe Salvo-Cozzo di Pietraganzili (Agrigento, 1856-Palermo, 1925), studioso e catalogatore di manoscritti presso la Biblioteca apostolica vaticana, fu dal 1897 direttore della Biblioteca nazionale di Palermo. Su di lui cfr. G. DE GREGori, S. ButTò, Per una storia dei bibliotecari italiani del XX secolo. Dizionario bio-bibliografico 1900-1990, Roma, Associazione italiana biblioteche, 1999, pp. 157-158.

(14) Un'amicizia petrarchesca, cit., p. 169.

(15) Journal de Pierre de Nolhac après son mariage, cit., p. 252.

(16) Cfr. nota 6.

(17) [Versailles, 22 mars 1893].

(18) Journal de Pierre de Nolhac après son mariage, cit., p. 253. 
bon compte). Mais rien autre chose ne me semble établi jusque là. - Je regrette bien de n'avoir pas fait moi-même à Rome tout ce travail (j'avais tant de mss. à voir en peu de temps!), vous savez qu'il n'est pas dans mes habitudes de me contenter d'à-peu-près en ces matières. Les simples confrères ne font pas toujours de bonne besogne quand ce ne sont pas des amis ${ }^{19}$.

La solidarietà della scuola era motivata soprattutto dai modi con cui la polemica era stata impostata, tant'è vero che Nolhac venne prontamente informato delle verifiche che si stavano conducendo in merito alla obiezione forte di Salvo-Cozzo:

Solerti m'écrit que Ferrari a collactionné le ms. 3195 et que les observations de S. Cozzo sont en général exactes. C'est l'édition de 1472 qui a été faite sur l'autographe. Je ne sais comment expliquer l'attitude de Bembo. - «Dallui dove bisogno è stato riveduto e racconosciuto ${ }^{20}$. C'est trop vrai. Ce sera à vous, son biographe, de nous en dire votre parere. De coeur ${ }^{21}$.

Così quando Giovanni Mestica «palesò con garbo le affrettate conclusioni del Nolhac $[. .$.$] ponendo la questione - senza le intemperanze del Salvo-Cozzo in termi-$ ni chiari» ${ }^{22}$, il Francese espresse la sua soddisfazione:

L'article de Mestica ${ }^{23}$ m'a paru remarquable surtout dans l'explication du rôle des éditeurs de 1501. Tout est à accepter dans le travail, sauf le passage sur l'Inventaire de la Vaticane, qui n'était point accessible, vous le savez comme moi d'expérience, aux travailleurs du dehors ${ }^{24}$.

La simpatia di Cian, sinceramente corrisposta, era rafforzata da una reciproca stima scientifica ed era ispirata ad una visione per certi versi assai simile. Basta leggere quanto, nel 1909, Nolhac scrive al corrispondente, in procinto di trasferirsi da Pisa a Pavia, ricevendo il volume offerto dai Suoi scolari dell'Università di Pisa ${ }^{25}$ :

Je vous ai dit, je crois, avec quel plaisir j'avais reçu le beau volume, magnifique témoignage de l'affection et de la reconnaissance de vos élèves pour un maître déjà illustre. Tout cela montre que nous vieillissons. Notre vie, du moins, n'a pas été inutile, et la vôtre surtout, cher ami, a bien servi la vérité, la science et la Patrie ${ }^{26}$.

Altrettanto eloquente un articolo, comparso sulla «Gazzetta del Popolo» in occasione della sospirata elezione di Nolhac all'Académie, nel 1922. Qui Cian gli riconosce, cosa rara per lui, una «triplice armatura d'erudizione, di scienza e di molteplice cultura - paleografia compresa» e si augura che egli possa accettare la direzione dell'École Française di Roma, in cui «fu missionnaire negli anni più felici della sua vita $\gg^{27}$. Lo stesso Nolhac avrebbe di lì a poco provveduto a disilluderlo, indicando un successore convincente nella persona di André Pératé («un des derniers élèves

(19) [Versailles, 22 mars 1893].

(20) Sul significato di questa frase cfr. G. MEsTi$\mathrm{CA}, \mathrm{Il}$ "Canzoniere" del Petrarca nel codice originale a riscontro col ms. del Bembo e con l'edizione aldina del 1501, «Giornale storico della letteratura italiana», XI (1893) p. 332.

(21) [Versailles, 15 mai 1893].

(22) G. Frasso, Appunti sul Petrarca Aldino del 1501, cit., p. 319.

(23) G. Mestica, Il "Canzoniere" del Petrarca nel codice originale a riscontro col $\mathrm{ms}$. del Bembo e con l'edizione aldina del 1501, cit., pp. 300-334.

(24) [Versailles, 7 juin 1893].

(25) A Vittorio Cian i suoi scolari dell'Università di Pisa (1900-1908), Pisa, Tip. ed. cav. F. Mariotti, 1909.

(26) Versailles, 30 juin 1909. L'apprezzamento di Nolhac nei confronti di Cian era sempre stato espresso in modo assai lusinghiero. A Novati che gli annunciava «una diligente recensione de' suoi Corrispondenti d'Aldo, dovuta [...] al nostro buon Cian» Nolhac rispondeva: «J'attends impatiemment l'article de notre excellent ami Cian; il y a toujours à apprendre et son amabilité s'y montre toujours de façon charmante», Un'amicizia petrarchesca, cit., pp. 64-65.

(27) V. Cian, Pierre de Nolhac, «Gazzetta del Popolo», 15 agosto 1922 . 
de G.B. De Rossi, dont la traduction de la Divine Comédie est sous presse» ${ }^{28}$ ), e spiegando le ragioni di una rinuncia dettata dalla volontà di «ne pas trop surcharger sa biographie, si l'on veut en prolonger la durée...»:

J'ai reçu [...] le bel article de la Gazzetta del Popolo ${ }^{29}$.

L'auteur m'a comblé, et j'apprécie plus encore les avantages de ma récente «immortalité», en voyant qu'elle m'apporte de telles marques d'estime et d'amitié. L'amitié surtout m'est précieuse, même si la bienveillance l'aveugle et lui fait exagérer mes mérites.

Vous n'exagérez point, en tous cas, bien cher ami, la solidité de mes sentiments pour l'Italie, et l'exhortation, si éloquente, de votre article, serait faite pour me décider à accepter cette lourde et glorieuse charge de la direction de l'Ecole de Rome.

Hélas! Si les objections accessoires se résolvent aisément dans mon esprit, par l'idée de remplir un devoir utile au moment présent, il est une objection plus grave qui se dresse devant ce project. Ma santé, délicate depuis plusieurs années a fléchi beaucoup en ces derniers temps, et le médecin aussi, qui me soigne à Royat, me détourne d'assumer les obligations fatigantes de la fondation. Ma famille, mes amis, sont unanimes à me conseiller le repos, qui me permettra de travailler encore par la plume, et peut-être par la parole, à servir les causes qui nous sont chères.

Bien que le Ministre de l'I.P. m'ait donné jusqu'au mois d'octobre pour me décider, je lui ai indiqué la décision qui s'impose.

Ah! Si j'était plus jeune seulement de cinq ou six ans, avec quelle joie j'irais achever ma vie parmi mes amis italiens et les souvenirs de ma jeunesse! C'est un regret profond; mais il faut savoir prendre son parti' .

Cian dovette trovare in quella risposta l'ennesima conferma di quanto aveva intuito della natura di Nolhac molti anni prima, in un «bel giorno del $1892 »^{31}$, quando l'amico, ritiratosi dalla Bibliothèque Nationale e rinunciando per sempre alla carriera di professore, era diventato direttore dei musei di Versailles ${ }^{32}$. «Un altro passato - sono parole di Ferdinando Neri - lo aveva chiuso nel suo fascino» ${ }^{33}$ lasciando che Petrarca, perso ogni interesse filologico e testuale, diventasse oggetto solo della sua «anima di poeta e artista», «tanto discreta da non invadere mai il campo assegnato all'opera del critico e dell'erudito» ${ }^{34}$. Addirittura, fa notare Billanovich, «prima ancora di licenziare Pétrarque et l'Humanisme aveva fornito il primo libro delle sue nuove funzioni e della sua nuova maniera: La reine Marie Antoinette ${ }^{35}$ preceduto l'anno prima da un più modesto Le château de Versailles.

Se le cose stanno come si è appena intravisto, non stupisce allora più di tanto il non trovare nel carteggio il minimo accenno alle furiose polemiche petrarchesche che, fra il 1930 e il 1931, coinvolsero Calcaterra, Croce e Cian ${ }^{36}$, nonostante l'invio («Spero che ti piacerà: è robba $[$ sic $]$ per te») delle poésies sull'«écolier d'Avignon ${ }^{37}$

(28) Royat, 20 août 1922.

(29) V. Cian, Pierre de Nolhac, cit.

(30) Royat, 20 août 1922.

(31) V. Cian, Pierre de Nolhac, cit.

(32) Il 21 aprile 1893 Nolhac scriveva a Cian: «Vous n'avez donc pas reçu la lettre fermée où [...] je vous demandais [...] par retour de courrier, sur cartoline la traduction en italien de la formule suivante pour mon Musée. I Avis. Les guides et interprètes offrent leurs services aux visiteurs sous leur seule responsabilité, le public est prévenu que l'Administration n'assume aucune responsabilité en ce qui concerne la fixation du taux de la rémunération qu'ils croient devoir réclamer. I Je renouvelle ma demande en la pressant».
(33) F. Neri, Pierre de Nolhac, «Nuova antologia», 16 febbraio 1936, p. 448.

(34) V. Cian, Pierre de Nolhac, cit. 323.

(35) G. Billanovich, Nolhac e Petrarca, cit., p.

(36) Né si trova cenno alle postille presunte bembiane su cui stava lavorando Cian: per entrambe le questioni cfr. C. Allasia, «Petrarca for ever!»: «interpellanze petrarchesche» nel carteggio CalcaterraCian, in Petrarca in Piemonte: Griselda, il Po..., Atti del Convegno (Torino-Saluzzo 2-4 dicembre 2004), a cura di P. Pellizzari e S. Re Fiorentin, che costituisce il n. 6 (2004) di «Levia Gravia», pp. 43-80.

(37) P. DE NolHac, L'écolier d'Avignon (Vaucluse 1352), «Revue des deux mondes», 1 janvier 1931 
[...] uscite il $1^{\circ}$ gennaio nella "Revue des deux mondes" $»^{38}$. Qui Nolhac «s'imagine écolier d'Avignon, suivant Pétrarque, et écoutant ses leçons, à Vaucluse, puis séparé de lui lorsque l'Italie rappelle à elle son poète» ${ }^{39}$.

Leggendo la prima di queste liriche, dal titolo La rencontre, Cian dovette notare una curiosa coincidenza:

Je t'avais rencontré dans la maison du Pape,

Maître François! [...]

Muet et recueilli, tu lisais à l'écart.

C'était la haute salle où de secrets passages

Conduisent les savants près des livres des sages

$[\ldots]$

Et l'heure ainsi passait et coulait après l'heure,

Et l'exact sablier se retournait souvent

Sans ralentir l'essor de ton labeur fervent

Mais, comme allait vers toi mon cœur prêt à te suivre

Tu me vis, tu souris et tu fermas le livre.

La scena si svolge «dans la maison du Pape» e richiama, per contrasto, un aneddoto, narrato nei Souvenirs d'un vieux romain, che aveva colpito anche Cian: un giorno, al passaggio di Leone XIII, solito attraversare le sale della Biblioteca Vaticana per recarsi nei giardini, «les catholiques s'étaient agenouillés; les protestants saluaient profondément», solo il più illustre fra i presenti, Theodor Mommsen, «n'avait pas levé les yeux; il affectait ne rien entendre, et ne voulait rien voir $\gg^{40}$. Non così Petrarca, che abbandona i suoi libri e conduce Nolhac «fier d'être vu marchant à son coté» per le strade di Avignone mentre «le soir tombait sur la cité».

Cian sapeva cogliere queste tracce di scrittura, così come, probabilmente, non si era ingannato sul lento allontanamento di Nolhac dagli studi italiani, non certo immediatamente percepibile o confessato dall'italianisant che sognava, nel 1892, di creare un «"Giornale storico" français», andando incontro a molte difficoltà («Mais, le croirez-vous? L'éditeur a reculé: la revue ne se fondera pas; on craint qu'elle n'ait pas de public et ne puisse pas vivre! Proprio così. Je ne sais à présent quand ce projet pourra se reprendre et j’en suis désolé!» $\left.{ }^{41}\right)$, superate con grande sforzo solo due anni dopo («Nous allons enfin avoir notre "Giornale Storico" pour 94 grâce à une Société d'histoire de la littérature française dont les cotisations feront vivre la revue» ${ }^{42}$ ).

Ben presto però Nolhac mette in chiaro, con una punta di civetteria, che l'eclettica ricerca di Cian «transfuge» dal «beau domaine de la Renaissance» impegnato in «aventureux et féconds voyages en terra incognita», è «bien éloigné de ses études, de ses sympathies et de sa compétence», ma «lit avec joie» i suoi contributi, ricchi

(CI), pp. 173-183, poi confluito in Le rameau d'or, Paris, Plon, 1933.

(38) [Paris,] 22 décembre 1930.

(39) G.E. Broche, Quelques lettres de Pierre de Nolbac. Défenseur de l’amitié Franco-Italienne, Paris-Gênes, Alliance Française, 1938, p. 52.

(40) P. DE NolHac, Souvenirs d'un vieux romain, Paris, Plon, $1930\left(1922^{1}\right)$, p. 20. Su questo libro si può vedere A.-C. FAITROP-PORTA, L'école française de Rome et les «souvenirs romains» de Pierre de Nolhac, de Jérôme Carcopino et de Pierre Grimal, in La réception du latin du XIX siècle à nos jours, Actes du colloque d'Angers 23-24 septembre 1994, textes réunis par G. Cesbron et L. Richer, Angers, Université d'Angers, 1996, pp. 337-348. Si cfr. anche il cenno firmato da Cian (Vi. Ci.) nel Bollettino bibliografico del «Giornale storico della letteratura italiana», XLVIII (1930), pp. 162-163. Per i Souvenirs Nolhac avrebbe ricevuto l'alloro in Campidoglio il 21 aprile 1931, con la seguente dedica «Palatinae laurus | quas | alma mater genuit | ROMA | Petro de Nolhac | filio urbis amantissimo | universali Latinitatis | gaudio DD».

(41) Versailles, 4 octobre 1892.

(42) [Versailles, 7 juin 1893]. 
dell'«aimable esprit [qui] se peint si bien dans votre style» ${ }^{43}$, correggendo anche talvolta qualche buffo errore:

(P[age] 13 de votre article, 1[igne] 3, il faut lire «il Leclerc, deputato di Maine e Loire», Maine et Loire est le nom du département ${ }^{44}$. P[age] 20, il manque certain[emen]t une syllabe au vers important de Legouvé mis en italique). Vous voyez, par ces observations, avec quel soin je vous ai lu, puisque j'ai relevé ces infiniment petits. Comme vous m'en signaleriez davantage dans mes études italiennes, hélas! $!^{45}$

\section{Lo $\ll$ Spirto gentil $»^{46}$ gli ispira una delle rare lettere in italiano:}

ho avuto grandissimo piacere a leggere la sua ottima dissertazione sull'eterna questione dello «Spirto gentil». Come è viva, piacente, sottile, la sua discussione, chiaro e piccante il suo stile, seducente la sua congettura. Veramente, con tutta la sua modestia, credo che habbia fatto la luce nelle limiti possibili, e oramai io che non aveva opinione, battaglierò per messer Cola. $\mathrm{E}$ sarà piacere di trovare arsenale tanto ben fornito nel lavoro di sì caro amico ${ }^{47}$.

Così vengono accolti con grande interesse lo «spécimen» e l'annuncio «de vos grands travaux italo-espagnols ${ }^{48} \gg$, e la quasi coeva Memoria gesuitica ${ }^{49}$, «travail si curieux, si neuf si impartial», che Nolhac promette di comunicare a «M. Morel-Fatio, secrétaire de l'Ecole des Chartes (rue des Francs-Bourgeois Paris)», assicurando a Cian che «il serait placé en mains très compétentes et très curieuses des relations italo-espagnoles $»^{50}$. Dante suscita il suo interesse ( $«$ J'attends votre Veltro ${ }^{51}$ et en parlerai $\gg^{52}$, anche se, dopo averlo letto, l'idea di un «compte-rendu sur une matière si éloignée de mes études» ${ }^{53}$ lo spaventa e promette una «annonce de Chronique». Bernardo Bembo è argomento maggiormente gradito:

Merci, cher ami, de l'envoi de votre travail sur Messer Bernardo ${ }^{54}$. C'est comme toujours vraiment plein de suc et de nouveauté. L'ode sapphique [sic] de la p. 12 est fort curieuse et manque aux Mille poeti de C. Del Balzo ${ }^{55}$. Tout ce qui touche votre Bembo m'intrigue fort, surtout écrit par vous ${ }^{56}$.

La lontananza dagli studi di Italianistica e la drammatica carenza di tempo diventano una valida argomentazione per non partecipare alle miscellanee in onore dei maestri della scuola storica: «pour les Mélanges Graf je ne compte pas collaborer, n'ayant absolument pas le temps de mettre en pied la moindre chose étrangère à mes

(43) [Versailles, 30 octobre 1895].

(44) Cian in effetti aveva scritto «I deputati Leclerc, De Maine e Loire (che forse erano appunto i membri della commissione eletta l'anno prima)», V. Cian, Per la storia del sentimento e della poesia sepolcrale in Italia ed in Francia prima dei "Sepolcri" del Foscolo, «Giornale storico della letteratura italiana», X (1892), pp. 205-235.

(45) [Versailles, 4 octobre 1892].

(46) V. CIAN, Ancora dello "Spirto gentil" di messer Francesco Petrarca, «Atti della Regia Accademia delle Scienze», vol. XXVIII (1892), pp. 882-928.

(47) [Versailles, 12 settembre 1893].

(48) V. Cian, Italia e Spagna nel sec. XVIII. Giovanni Battista Conti e alcune relazioni letterarie tra l'Italia e la Spagna nella seconda metà del Settecento. Studii e ricerche. Torino, Libr. scient-lett. S. Lattes, 1896.

(49) V. Cian, L'immigrazione dei Gesuiti spagnuo- li letterati in Italia, «Memorie della Reale Accademia delle Scienze», Torino, XLV (1894-95), pp. 1-66.

(50) [Versailles, 28 juin 1895].

(51) V. Cian, Sulle orme del veltro: studio dantesco, Messina, Principato, 1897.

(52) Versailles, lundi 17 [août 1897].

(53) [Versailles, 27 novembre 1897].

(54) V. Cian, Per Bernardo Bembo. Le relazioni letterarie, $i$ codici e gli scritti, «Giornale storico della letteratura italiana», XVI (1898), pp. 49-81. L'ode saffica a cui Nolhac fa riferimento subito dopo è da leggersi alle pp. 60-61.

(55) Poesie di mille autori intorno a Dante Alighieri, raccolte ed ordinate cronologicamente con note storiche, bibliografiche e biografiche da C. Del Balzo, Roma, Tip. Forzani e C. Edit, voll. 115, 1889-1909.

(56) [Versailles, 19 février 1898]. 
études actuelles» ${ }^{57}$. Il vero motivo, o uno dei motivi, emerge però alla richiesta del contributo per la miscellanea Renier. «J'attends la circulaire Renier et ferai le possible» promette Nolhac in un primo tempo, pur non mancando di ribadire «que mes études sont bien loin aujourd'hui des vôtres» ${ }^{58}$. Qualche mese dopo scrive «j'ai reçu [...] votre appel affectueux pour Renier ${ }^{59}$, e afferma subito che potrebbe tentare di «faire quelque chose à cause de vous, plus qu'à cause de celui» che, constata amaramente, «j'ai perdu de vue depuis si longtemps» ${ }^{60}$.

Forse ha contribuito a costruire questa esibita lontananza anche la recensione alla seconda edizione del Pétrarque et l'Humanisme, comparsa sulla «Cultura» ${ }^{61}$ a firma di Remigio Sabbadini (sostituitosi all'ancora una volta esitante Novati), «où il a relevé utilement bien de taches dans mon livre, sans reconnaître assez peut-être l'effort fait pour le remettre au courant $\gg^{62}$. Il libro gli era molto caro ed è l'unico caso in cui dà notizia a Cian delle varie occasioni di traduzione ${ }^{63}$, fasi di riscrittura ${ }^{64}$ e revisione $^{65}$.

Ma accade qualcosa di imprevedibile: nel giro di un decennio il mondo da cui Nolhac sta compostamente prendendo le distanze scompare.

J'ai appris avec stupeur et émotion la douloureuse nouvelle! Merci des détails que vous m'avez donnés, cher ami, j'ai écrit aussitôt à la pauvre veuve, tant à plaindre avec sa nombreuse famille! Je regretterai toujours le bon et loyal ami, l'excellent compagnon de travail que fut Solerti ${ }^{66}$.

La morte di Angelo Solerti, coautore nel 1890 di Il viaggio in Italia di Enrico III e considerato nel Journal uno «des amis très chers [...] hors de la frontière, que j’aime beaucoup mieux des amis français» ${ }^{67}$, sembra quasi far presagire quello che sta per succedere: «Resserrons nous bien fidèles, cher ami, à mesure que s'éclaircissent les rangs de notre jeunesse ${ }^{68}$, scrive angosciato Nolhac, pur non potendo immaginare che si tratti del primo di una lunga serie di lutti. Non scompariranno soltanto Pascoli e Fogazzaro, per i quali Nolhac mostra grande ammirazione ${ }^{69}$, ma la cerchia dei savants italiens con cui è in contatto sarà decimata:

(57) [Versailles, 10 avril 1902].

(58) [Versailles, 1 avril 1910].

(59) Scritti varii di erudizione e di critica in onore di Rodolfo Renier, Torino, Bocca, 1912.

(60) [Versailles, 14 février 1911].

(61) 26 (1907), pp. 347-350.

(62) Un'amicizia petrarchesca, cit., lettera del $1^{\text {er }}$ décembre 1907, p. 303. «Sabbadini lasciò stridere le asprezze del suo temperamento e difese la tesi meschina [...] che nel ritrovamento dei testi classici il Petrarca sia rimasto molto al di sotto del Boccaccio e dei minori contemporanei; ma [...] anche elencò le inesattezze mantenutesi in questa seconda edizione» (G. Billanovich, Nolbac e Petrarca, cit., p. 324). Se a livello nazionale «questa recensione passò inavvertita», tuttavia non sfuggì a Nolhac, e, secondo Alberto Brambilla, «dettò quasi emblematicamente la fine del carteggio con Novati» (Un'amicizia petrarchesca, cit., p. XL).

(63) «On publiera en automne une traduction partielle de P. et l'Hum. à Boston! Edition de bibliophile que je ne pourrai envoyer hélas à mes amis. Il n'y aura d'ailleurs, rien de nouveau. Je vous envoie la feuille initiale que je viens de recevoir d'Amerique», [Versailles, 26 août 1904].

(64) «Je suis occupé, en ce moment, à le revoir entièrement en vue d'une nouvelle édition et j'aurai sans doute à vous interroger sur quelques doutes. Dès à présent si vous avez des corrections à m'indiquer [...], je serai heureux de les recevoir. Bien entendu, j'utilise vos "Nugellae vulgares" etc.», [Versailles, 28 octobre 1905]; «Mon livre [...] paraîtra très augmenté en 2 vol. au cours de l'an prochain, et à propos duquel j'aurai sans doute occasion de vous écrire encore», [Versailles, 27 décembre 1905], «Je prépare pour le début du 1906, la réimpression corrigée du augmenta de P. et l'Humanisme. J'y citerai votre nom plus d'une fois. Toute correction ou addiction qui vous viendrait à la pensée serait la bienvenue», [Versailles, 15 avril 1905].

(65) «Petrarca cammina adagio adagio», [Versailles, 13 febbraio 1906], «P. et l'Hum. chemine lentement, et le vol. I est fini; le tout paraîtra en mars», [Versailles, 26 décembre 1906].

(66) [Versailles, 19 janvier 1907].

(67) Journal de Pierre de Nolhac après son mariage, cit., p. 274.

(68) [Versailles, 19 janvier 1907].

(69) Il 30 aprile del 1898, in occasione del suo viaggio a Messina, Nolhac chiedeva al corrispondente: «Pascoli est il à Messina? Je serai charmé de faire sa connaissance» e in calce a una lettera del 12 aprile 1912, 6 giorni dopo la morte del poeta, annotava: «Povero Pascoli!». A proposito di Fogazzaro 
Je lis dans notre cher «Fanfulla» votre bel article sur D'Ancona ${ }^{70}$. Quel admirable maitre, et comme vous décrivez bien sa bibliothèque où j'ai passé quelques bons moments de causerie. Ici, q[uel]ques journaux ont rappelé son amitié avec Renan et G. Paris ${ }^{71}$.

Gli scarsi contatti con Rodolfo Renier rendono quasi inattesa la sua scomparsa, nonostante le penose condizioni degli ultimi mesi:

J'apprends avec une vive peine la mort du pauvre Renier. C'est une grande perte pour la science - et pour vous particulièrement, c'est un grand deuil d'amitié, après tants d'autres ${ }^{72}$.

Ma è l'improvvisa morte di Novati, quasi presagita pochi mesi prima («à ce propos, je vous demanderais, à l'occasion, de me donner de nouvelles de Novati. On m'a dit qu'il avait été fort malade, et de fait il n'écrit à personne» ${ }^{73}$ ), a impressionarlo profondamente: «La mort du pauvre Novati m'a fait une peine bien grande. Il y a 29 ans qui il m'ouvrait, avec le bon Renier, le "Giornale Storico" [...] !»

Graf, Renier e Novati, «nos chers disparus»" ${ }^{75}$,Vos chers morts illustres [qui] sont aussi un peu les miens» ${ }^{76}$, spostano il rapporto con $\mathrm{i}$ «savants italiens» su un piano fortemente emotivo: «nous voilà sur le seuil de la vieillesse et de la mort» ${ }^{77}$.

Forse per questo alla lontananza sempre più esibita sul piano scientifico e tematico, si accompagna un'attenzione profonda al rapporto personale nei confronti del corrispondente: Nolhac guarda con affetto non privo di lieve ironia al «votre cher monde "piccolo mondo moderno"» e raccomanda a ogni lettera di «embrasser les "bimbi" [i figli Gilda e Alberto] pour le vieil ami de leur père» ${ }^{78}$. Aveva visto il giovane Cian innamorarsi e, nel 1892, ricevendo le Candidature nuziali di Baldassarre Castiglione, chiedeva, a costo di passare per indiscreto: «Et vous même, quand vous-vous candidate? $\gg^{79}$, senza peraltro nascondere gli ostacoli che la situazione matrimoniale pareva porre a una vita di studi:

Tous nos enfants sont malades en ce moment et c'est bien du souci pour la maman qui vous envoie son bon souvenir, à son tour. C'est aussi une nouvelle perte de temps, pour moi, qui dois m'en occuper un peu. Foelix coelebs! ${ }^{80}$

Nel 1893 si era mostrato vivamente partecipe al lutto che aveva colpito i Cian: la morte prematura di Giorgio Trentin, marito della sorella di Vittorio, Italia, e padre di Silvio ${ }^{81}$ :

Nous prenons à votre douleur, ma femme et moi, et à celle de Madame votre sœur la part la plus vive et la plus amicale.

Comme les pauvres petits sont à plaindre aussi, et quels graves et nouveaux devoirs surgissent pour vous, avec le cœur excellent que nous vous connaissons, de cette situation nou-

si legga invece la lettera del 19 gennaio $1907:$ «Nous recevons avec enthousiasme votre cher et grand Fogazzaro. Sa conférence a été fort belle et fort brave» $\mathrm{e}$ in una lettera a Gaston Broche del 1929 ricordava «Fogazzaro, que j'ai reçu chez moi à Versailles, et dont j'ai été l'hôte dans sa maison de Vicence», Quelques lettres de Pierre de Nolhac, cit., p. 44. Per le tracce di questo rapporto nei suoi scritti rimando a A. Brambilla, Appunti su alcune lettere di Pierre de Nolbac al Fogazzaro, in Antonio Fogazzaro. Le opere e $i$ tempi, Atti del convegno internazionale (Vicenza 27-29 aprile 1992), a cura di F. BANDINI e F. FinotTi, Vicenza, Accademia Olimpica, 1994, pp. 287-304.

(70) V. CiAn, Alessandro D’Ancona, «Fanfulla della Domenica», 15 novembre 1914

(71) [Versailles, 22 novembre 1914].

(72) Château de Versailles, 13 janvier 1915.

(73) Ibidem.

(74) [Versailles, 19 janvier 1916]

(75) Paris, 20 décembre 1920.

(76) [Versailles, 21 novembre 1918].

(77) [Versailles, 19 janvier 1916].

(78) Londres, 25 avril [1900].

(79) [Versailles, 30 octobre 1992].

(80) [Versailles, 16 avril 1893].

(81) Per qualche cenno sul rapporto fra il giovane Silvio Trentin e lo zio cfr. C. Allasia, Prime spigolature dal Fondo Cian, cit., pp. 12-19. 
velle! Nous vous plaignons du fond du cœur de l'épreuve nouvelle que vous traversez et je vous embrasse plus affectueusement que jamais ${ }^{82}$.

L'amico francese tentava di distrarre Cian che, «au retour de son triste voyage» a San Donà, aveva avuto «le loisir d'écrire encore» «des lignes», e si affrettava a promettergli di «ne point tarder à aller vous serrer la main, et causer de Pétrarque viva voce» ${ }^{83}$.

Nel 1898 avrebbe cercato per sé una parola di consolazione per la scomparsa, avvenuta il 10 luglio di quell'anno, del figlio François:

Merci [...] des affectueuses paroles avec lesquelles vous avez pris part à notre grand deuil. Je ne vous ai pas répondu sur le champ, parce-que j'ai vécu plusieurs semaines dans un grand désespoir. Cet enfant était le meilleur et le plus aimé de tous.

La pauvre maman surtout est démeurée accablée bien que sa foi chrétienne très agissante contribue à la soutenir. Sa santé s'en est même ressentie et elle ne va pas bien en ce moment. Mes deux grands garçons, 19 et 14 ans continuent à travailler assez bien. Nos fillettes qui me font penser à votre joie paternelle, sont notre meilleure consolation ${ }^{84}$.

Lui aveva per sé, ammette, una possibilità, negata alla moglie Alix: «Puis, j’ai le travail! J'ai entrepris la publication de ma grande histoire de Versailles et, pour activer le travail, j’ai renoncé cette année à faire une conférence sur la Renaissance».

Tornando a occasioni più liete, nel 1894 aveva operato attivamente nella cerchia degli amici francesi per diffondere la pubblicazione celebrativa delle nozze Cian-Sappa Flandinet, uscita, non solo a causa della mole, con notevole ritardo:

J'ai reçu hier seulement de Bergamo le magnifique volume ${ }^{85}$. J'y occupe une bien modeste place et je regrette d'avoir pu vous offrir si peu mais votre amitié veut bien s'en contenter et c'est moi qui la remercie. Je porterai cette semaine le vol. chez M. Léopold Delisle et à M. Meyer (G. Paris est déjà en vacances et n'aura le sien qu'en nov.bre). La «Romania» en parlera certain[emen]t et je me charge de la «Rev. Crit.» et d'un mot dans la «Rev. d'hist. Littér.». J'attends avec impatience les compte-rendus italiens qui répéteront quelquefois de plus votre nom aux échos de gloire uni au nom qui vous est $\operatorname{cher}^{86}$.

La confidenza epistolare è alimentata anche da alcune felici occasioni d'incontro. Nel 1894 i coniugi Nolhac sono ospiti dei Cian a Torino:

J'aurai une visite artistique à faire via Lagrange. Puis, nous serons tous aux amis - et particulièrement à l'ami Cian. La sequestrazione! Mais nous ne demandons pas mieux! Probablement nous pourrons rester jusqu'au lendemain à la même heure: mais ce n'est pas encore bien décidé. Peut-être le départ subira-t-il un certain retard, si une de nos petites filles, qui tousse un peu, paraissait devoir être malade. Je vous préviendrais tout de suite de ce retard, qui heureusement, parait peu probable. Merci, cher ami, de votre joie amicale si vivement exprimée. La mienne n'est pas moindre, croyez-le bien. De coeur ${ }^{87}$.

Di una visita in Sicilia («me voici Sicilien depuis hier soir» $\left.{ }^{88}\right)$ accennerò più avanti. Da parte sua Cian è ricevuto, più di una volta, a Versailles dove «lo studioso ap-

(82) [Versailles, 3 mai 1893].

(83) [Versailles, 15 mai 1893].

(84) [Versailles,] 28 octobre [1898].

(85) Nozze Cian-Sappa Flandinet, 23 ottobre 1893,

Bergamo, Istituto italiano d'arti grafiche, 1894.
(86) Versailles, 11 juillet 1894.

(87) Versailles, [6 mars 1894].

(88) Taormina, Albergo Vittoria, ven., [30 avril 1898]. 
passionato della nostra Rinascita, figura esile d'artista amabilmente signorile, godeva di fare gli onori di casa» ${ }^{89}$ :

C'est en rentrant ici, cher ami, [...] que je trouve l'heureuse nouvelle de votre passage à Paris. De grâce, accordez à l'amitié 24 heures à Versailles, il nous sera si facile de vous recevoir dans le Château! Et votre lit est déjà préparé ${ }^{90}$.

Il 1909 darà occasione ai Cian di «recevoir [...] à Ceres avec tant d'amitié...» ${ }^{91}$ Marie Louise, la diciottenne «fille aînée» di Nolhac, in vacanza in Piemonte.

Meno sereni gli incontri successivi: a Roma nel 1918, per tenere un corso su invito del rettore, il matematico Alberto Tonelli, Nolhac si rallegra delle notizie positive che arrivano dal fronte, pur aggiungendo subito «Déjà les attaques aériennes recommencent sur Paris, et préludent aux autres»:

J'ai quitté Rome à l'heure où arrivaient du Piave les magnifiques nouvelles. J'ai partagé une fois encore les grandes émotions de l'Italie et vu, de mes yeux fraternel, le spectacle réconfortant de vaillance. C'est notre tour, à présent, d'attendre le choc de l'ennemi; puissions-nous faire d'aussi belle besogne que l'armée de Diaz! ${ }^{92}$

Nelle ultime lettere che abbiamo scorso la dimensione erudita o quella privata e familiare sono insidiate dall'incombere drammatico della storia, a cui Nolhac reagisce sempre con stupefatto dolore. È un atteggiamento riscontrabile fin dal 1893 quando commenta con Cian gli scontri di Aigues Mortes:

Ces faits d'Aigues mortes sont douloureux hélas! - mais d'ordre tout à fait local et ouvrier. Aussi, personne en France (pas même moi) n'a rien compris aux manifestations gallophobes qui ont éclaté à Rome et partout. C'est l'indice d'un état d'esprit que nous ne connaissons pas, heureusement dans les classes cultivées des nations. Ces classes, j'en ai la ferme espérance, feront la réconciliation complète avant la fin du siècle ${ }^{93}$.

Sul suo Journal constata il «dernier froissement entre la France et l'Italie qui ont amené $[. .$.$] avec plusieurs de mes amis de là-bas un échange de lettres émues» { }^{94}$, e in una lettera in italiano, di poco successiva:

Io respiro un poco, poiché è finita la settimana dolorosa del viaggio nella nostra Milano e la nostra Venezia. Il mio popolo ha visto odiosa provocazione dove vedo piuttosto colpa purtroppo senza rimedio... Dio salvi i popoli latini! $!^{95}$

Nel $1901^{96}$ esprime agli amici italiani tutta la sua vicinanza per l'attentato a Umberto I e mostra di apprezzare la commemorazione inviatagli da Cian, duramente censurata, con argomentazioni diverse ma affini, da Croce e da Graf ${ }^{97}$.

(89) V. Cian, Pierre de Nolhac, cit.

(90) Château de Versailles, $77^{\text {bre }}$ [1901].

(91) 12 août 1916.

(92) Versailles, Vendredi [28 juin 1918]. Nolhac ricorderà questo episodio in un lettera di cinque anni più tardi: «Vous vous rappelez en quelles circonstances tragiques de la guerre nous nous sommes rencontrés, en 1918, pour la dernier fois», $\mathrm{Pa}$ ris, 16 octobre 1923 .

(93) [Versailles, 25 août 1893].

(94) Journal de Pierre de Nolhac après son mariage, cit., p. 391.
(95) [Versailles, 12 settembre 1893].

(96) Il 13 luglio 1900 scriveva a Cian: «nous apprenons avec émotion et douleur le deuil qui frappe votre cher pays. Mon coeur s'unit au vôtre en ce cruel jour et fait des voeux pour la prospérité de l'Italie et la gloire du règne nouveau».

(97) In memoria di Umberto I di Savoia. Discorso letto il giorno 12 agosto 1900 nella chiesa parrocchiale di Ceres, Torino, tipogr. S. Giuseppe degli Artigianelli, 1900. Per la reazione di Graf si cfr. le Lettere a Vittorio Cian, a cura di chi scrive, Firenze, Le lettere, 1996, p. 152. Croce confutò invece la 
J'ai reçu en son temps et lu avec émotion votre belle commémoration du pauvre Roi. vant $^{98}$.

Le fait de la dire, vous laïque, en une église avait déjà quelque chose de noble et d'émou-

Pur essendo entrambi uomini d'ordine, i due corrispondenti mostrano alcune differenze: Nolhac condanna il J'accuse zoliano senza esitazione e con maggior franchezza di quanto avesse fatto con Novati:

Nous sommes ici dans la fièvre du procès Zola. Ni moi, ni mes amis ne sommes au nombre des partisans de cet écrivain qui a écrit des abominations et sera justement puni. C'est vous dire que tant de manifestations venues d'Italie (et sans doute généreuses d'intention) nous ont fait de la peine ${ }^{99}$.

È chiarissimo che Nolhac non apprezza le mobilitazioni italiane in favore dell'autore di Nana e cerca di convincere Cian, in occasione di una rapida visita in Sicilia di ritorno da «son voyage d'Orient»:

J'aurai au moins le grand plaisir de passer avec vous, cher ami, quelques bonnes heures. - Nous causerons de l'affaire Dreyfus - et je vous montrerai qu'elle n'est pas aussi simple, au point de vue de la critique historique, que ne l'imaginent les naïfs rédacteurs du «Corriere»; - mais nous causerons surtout de nos chères études et de notre vieille amitié ${ }^{100}$.

La più drammatica incursione del presente, la Grande Guerra, getta le basi di quella che sarà la cocente e dolorosa delusione politica di Nolhac:

Nous sommes dans une tourmente terrible; mais avec la volonté de vaincre et la certitude que c'est le combat pour la civilisation qui se livre contre les Barbares. Oh, cher ami, poussons-le ensemble notre vaillant cri: Fuori i barbari! [... Le sort du Louvain, du Malines, nous apprend ce qui nous attend, mais tous nous avons fait d'avance le sacrifice de nos vies, et nous sommes sûrs que ce n'est pas la France qui périra ${ }^{101}$.

Per un uomo che si trova in questa «gigantesque lutte», «avec fils et gendre à la frontière et de graves inquiétudes pour le trésor national et universel, dont il a la responsabilité» ${ }^{102}$, il mestiere delle lettere perde significato:

Mais nous n'avons plus de pensée pour les Lettres... L'heure est si grave pour nous ${ }^{103}$.

L'entrata in guerra dell'Italia («Je crois à la "plus grande Italie”, qui va fixer ses destinées, tout en travaillant à la libération du monde» $\left.{ }^{104}\right)$, celebrata da Nolhac con una raccolta di poesie («l'expression lyrique de ma fidèle pensée»), è vista come una conferma della vicinanza e dell'affinità con la Francia:

commemorazione in una lunga riflessione trascritta ora in B. Croce-V. Cian, Carteggio, a cura di chi scrive, Napoli, Istituto Italiano per gli Studi Storici, in corso di stampa, lettera $\mathrm{n}^{\circ} 109$.

(98) [Versailles, 13 septembre 1900].

(99) [Versailles, 19 février 1898].

(100) [Versailles, 18 mars 1898].

(101) [Versailles, 31 août 1914].

(102) Versailles, 2 avril [1915].

(103) [Versailles, 22 novembre 1914]. Si cfr. l'affermazione di Cian in una lettera a Croce: «in questi mesi di passione e di tormento io non ho il pensiero ad altro che a questa nostra guerra, che mi tiene in una febbre continua dello spirito, mi toglie quella serenità che è necessaria agli studî, al punto da farmi considerare come povere cose quegli studî stessi che un tempo erano uno dei conforti maggiori della mia vita», B. Croce-V. Cian, Carteggio, cit., n॰302.

(104) Versailles, 2 avril [1915]. 
Comme je pense à vous en ces jours d'émotions sublime et tragique! Votre pays a donné au monde, pendant ce mois historique, le plus admirable exemple de sagesse et d'enthousiasme.

Cette unanimité d'un peuple aujourd'hui acquise par le plus terrible des sacrifices est un spectacle d'une incomparable beauté. S'il y avait, parmi nous français, des sceptiques à l'égard de l'Italie, je vois que leurs yeux se sont ouverts et qu'il sont forcés, eux aussi, de vous admirer. L'épreuve sera dure, mais quels horizons s'ouvrent à vous! Cette heure est celle dont parle le poète nouveau: «Apri alla nostra virtù le porte del dominio futuro» ${ }^{105}$.

Et que de fois, j’ai mesuré comme un appel les vers de l'autre poète: «Sia gloria o fratelli! Non anche | l'opere del secol non anche è piena» ${ }^{106}$.

Quels sacrifices à vous personnel ou autour de vous, va vous demander la Patrie? Je les connais d'expérience; mais, grâce à Dieu, mes deux fils sont encore sains et saufs - quoique sur le front depuis tant de mois! ${ }^{107}$

Di fronte alle violenze commesse in Belgio («Ces horreurs, hélas!»), Nolhac constata che un mondo, il suo mondo, è irrimediabilmente perduto: «Quelles pages pour la Kulturgeschichte!». Il riscatto, ammesso che sia possibile, parte ancora una volta dalla garanzia offerta dal passato: «Heureusement que l'héroïsme de tant de vaillants, comme vos Garibaldi, relève l'honneur de notre temps avant l'humanité future» ${ }^{108}$.

Ai primi successi degli alleati, Nolhac intravede «les vastes espoirs qui s'ouvrent devant la race latine enfin vengée» e trova la forza di complimentarsi per «les victoires italiennes, l'admirable refoulement de l'ennemi sur le Trentin, la prise de Gorizia» che provocano «des joies profondes pour son cœur» e «une fois de plus, le rapprochent du vôtre» ${ }^{109}$. Tanto entusiasmo non cela il suo profondo dolore personale: il presente ha preteso il suo tragico tributo di sangue. L'amata «fille aînée» Louise, amica di Gilda Cian e ospitata anni prima a Procaria, è rimasta vedova: «Son cher mari a été tué devant Verdun» ${ }^{10}$ e lei «élève en Auvergne dans le deuil et dans le courage chrétien, ses quatre petits orphelins» ${ }^{111}$.

La condizione «d'avoir pas l'honneur de combattre et de servir au front», avvicina i due corrispondenti e li fa sentire «l'un et l'autre, emportés dans le tourbillon des événements où se jouent les destinées de notre patrie», determinandone la volontà «de faire un peu de bien, d'exercer un peu d'action utile», soprattutto attraverso $l^{\prime} \ll$ apostolat de plume et de parole» ${ }^{112}$.

Il 30 aprile 1917, sei giorni dopo Caporetto e un giorno dopo la caduta del governo Boselli, Nolhac cerca di scrivere parole di conforto:

Je vis ces jours douloureux dans une angoisse fraternelle! L'épreuve cruelle de l'Italie est notre épreuve à tous vos frères et vos amis à jamais ${ }^{113}$.

Anche dopo la destituzione, Cadorna, in visita a Versailles, resta «magnifique», e Nolhac gli mostra «à lui tout seul, les traités de Versailles». Sembra quasi che il generale appartenga a un passato che non riesce a influenzare, come dovrebbe, il presente:

(105) G. D'AnNunZIO, Ode al re giovane («apri alla nostra virtù le porte | dei domini futuri»).

(106) G. CARDUCCI, A una bottiglia di Valtellina del 1848. Nolhac aveva prefato nel 1891 la traduzione di Julien Lugol delle Troisièmes odes barbares, per i tipi di Lemerre e avrebbe ricordato affettuosamente il poeta nei Souvenirs d'un vieux romain $(A$ Bologne avec Carducci, pp. 73-86). Giuseppe Frasso rammenta che Carducci, «con la forza del suo prestigio», aveva autorevolmente ed erroneamente legittimato l'ipotesi di Nolhac relativa al Vat. Lat.
3195 (Appunti sul Petrarca Aldino del 1501, cit., pp. 315-16).

(107) Château de Versailles, 20 mai 1915, au matin.

(108) Château de Versailles, 13 janvier 1915.

(109) 12 août 1916.

(110) Ibidem.

(111) Château de Versailles, 10 octobre 1917.

(112) Ibidem.

(113) [Versailles,] 30 octobre [1917]. 
«ce repos d'un grand homme, au milieu de ces grands souvenirs, me laisse un souvenir ineffaçable». Ė, come Garibaldi, una «des belles figures à contempler dans cette guerre», «une des pages réconfortantes» ${ }^{114}$ da leggersi per evitare che lo scoramento possa prendere il sopravvento:

Nous sommes encore dans les mauvais jours, dans les mauvais mois, cher ami.

Mais vous avez comme moi, je suis sûr, l'indomptable espérance. [...] La Barbarie sera vaincue si nous nous défendons bien contre la trahison intérieure, qui fut si grave chez nous ${ }^{115}$.

E le «Pâques ensanglantées», «les plus terribles que le monde ait connues» ispirano sentimenti condivisi:

Il en va sortir - j'en ai la ferme confiance en Dieu - la résurrection du Droit dans le monde et la victoire si durement achetée de nos patries. Mais comment ne pas avoir le coeur serré à la partie qui se joue pour Amiens et pour Paris, alors que nos rues se remplissent à nouveaux de réfugiés douloureux - comme vous en avez, hélas! connus l'an dernier - et que l'on entend parfois tonner ici le canon de la Vallée de l'Oise! L'ennemi s'approche mais nous avons la ferme confiance qu'il n'y arrivera pas et que la semaine de Pâques nous apportera les premières promesses décisives. Je sais que vous pensez à nous comme nous pensons à vous - et que vous partagez nos angoisses et nos espérances. Tout n'est-il pas commun en cette guerre entre ceux qui combattent la Barbarie! ${ }^{116}$

Una delle lettere più elaborate e sentite è quella scritta nel gennaio 1919 sulla questione dalmata e vale la pena di leggerla per intero:

Pourquoi faut-il que les gens à qui notre union porte ombrage s'efforcent de la compromettre, pour ce qui n'est qu'un absurde malentendu? Cette histoire yougo-slave me désole, vous le pensez bien, mais plutôt par la façon trop émue dont je la vois juger en Italie. Ce n'est rien, en vérité, et il n'y a pas là de quoi altérer, même d'un nuage, le ciel de notre amitié de peuples. Je ne sais pas s'il y a à Paris deux journaux (en tout cas, il n'y en a pas trois) qui fassent la campagne slave. Les «Débats», qui ont de l'autorité littéraire, n'en ont aucune dans la politique et n'ont même pas convaincu leurs rédacteurs (dont je suis). C'est un bien mince résultat pour les Yougo-Slaves, après tant de campagnes de presse en Angleterre, tant de livres, de tracts, de brochures, de conférences [...]. L'opinion est ici plus que froide à l'égard de MM. les Croates et Slovènes. Ils ne comptent pour personne, malgré leur agitation. Ce n'est qu'en Angleterre qu'ils ont obtenu des résultats; et nous trouvons un peu attristant que ce soit à la France que s'en prennent les journaux de nos amis d'Italie alors que la France ne vous créera aucune difficulté à la Conférence, bien au contraire. Nous ne pouvons empêcher que nos soldats, accueillis par des soi-disants alliés, acclamés par des "Serbes" (!) leur fassent bonne figure. Il y aura toujours un membre de l'Institut (sur 250) pour représenter la thèse slave, parce-qu'il est président d'honneur de l'Académie de Zagreb! C'est le même, qui passe sa vie à médire des Polonais; mais les Polonais n'en rendent pas responsable la France. Il faut que les manifestations individuelles soient jugées avec dédain, car elles ne tirent pas à conséquence, n'étant suivies d'aucun résultat dans l'opinion.

J'aurais voulu que la question fût mieux posée dès l'origine. Je connais un peu cette question dalmate ("sur la mer où gouverna Venise"); mais mon pays l'a toujours ignorée profondément. On l'a enthousiasmé pour Trento et pour Trieste, et nous avons marché pour l'Italie de tout coeur. La Dalmatie et Fiume ont eu l'air d'apparaître après coup, et sans préparation, alors que la propagande croate faisait rage, très habilement appuyée sur la popularité de la Serbie. Nous ne pouvons donc pas nous enflammer pour la Dalmatie; et je le regrette, car personnelle- 
ment je voudrais vous voir occuper tous les points où vos tristes adversaires détruisent, par un vandalisme imbécile, le Lion de S. Marco (comme si le maillot des démolisseurs pouvait effacer l'histoire!). Je voudrais que vous possédiez, non seulement la maîtrise absolue de l'Adriatique, qui est votre droit chèrement payé par le sang de vos héros, - mais encore toutes les cités où la civilisation italienne domine par la tradition et l'existence d'une ancienne élite. Je voudrais que l'Italie fût l'éducatrice de ces peuples, encore médiocrement civilisés, que la nature lui a donnés pour voisins et qu'elle est mieux préparée qu'aucune autre nation à faire entrer dans le courant de la Civilisation Latine. Je voudrais surtout que quelques faits isolés, et en somme explicables, ne fussent pas l'occasion de renouveler les suspicions d'autrefois; et j'espère fermement que le président Wilson, le grand arbitre de Demain, éclairé par son voyage en Italie (Evviva Fiume!), saura "dire le droit" et reconnaitra nos efforts communs. Malgré l'affection et même l'admiration qu'on peut avoir pour les Serbes, qui ont eu leur part des charges communes, la France ne leur sacrifiera jamais, j'en suis convaincu, les droits bien établis de la Latinité représentée en Adriatique par l'Italie victorieuse ${ }^{117}$.

\section{In questo contesto La lettera ai Dalmati di D'Annunzio ${ }^{118}$ appare tutt'altro che opportuna:}

Nos journaux, qui ne connaissent rien à la question Dalmate, ont accueilli avec stupéfaction, et naturellement avec ironie, la lettre de D'Annunzio. Je ne l'approuve pas entièrement. L'allusion aux Pâques Viennoises, justifiée ou non, est exactement le contraire de ce qu'il faut dire aux Français. L'affaire a été déplorable. Pour moi, je comprends fort bien qu'il a voulu frapper un coup, pour secourir l'indifférence. Mais il ne pouvait pas trouver plus mal... ${ }^{119}$

Al momento del Trattato di Rapallo, che sancisce l'indipendenza di Fiume e la rinuncia italiana alla Dalmazia, Nolhac afferma di «partager ex imo corde l'émotion cruelle de ceux d'entre vous qui ont leurs yeux pleins de larmes fixés sur Fiume d'Italia. Mais, dans les ténèbres présentes, vous savez que reparaîtra bientôt plus brillante et plus fière l'étoile de la Patrie! $\gg^{120}$. D'altronde aveva espresso circa un anno prima la sua convinzione sulla necessità di una politica comune che non pagasse troppi dazi all'influenza degli Stati Uniti:

Les destinées de votre chère patrie, si intimement unies à celles de la nôtre - ce qu'elles finiront bien par comprendre l'une et l'autre - sont bien à une heure décisive. Chez nous, je vois avec plaisir l'action diplomatique s'orienter fortement vers l'Italie - et nous payons même fort cher cette attitude du côté de l'Amérique qui remet en question les pauvres satisfactions accordées par le traité à la France martyre. Il aurait mieux valu pour nous prendre plus tôt la partie de l'Italie dans l'Adriatique, je l'ai toujours pensé, vous le savez. Mais les torts ne sont-ils pas un peu partagés et sont-ils tous du côté de nos hommes d'État? L'essentiel - aujourd'hui - est qu'on puisse envisager un rapprochement qui nous est également nécessaire à tous dans l'ordre économique comme dans l'ordre politique ${ }^{121}$.

«Après la Signature de la Paix, a casa mia», Nolhac abbandona la «direction en chef du Musée du Versailles» ${ }^{122}$, il secondo dei luoghi della sua vita, quello che aveva voluto fosse raffigurato sul suo ex libris insieme a piazza San Pietro, e si trasferisce all'Institut de France, Musée Jacquemart-André: «Ici je suis au repos pour la fin de mes jours parmi de belles oeuvres d'art (c'est une sorte de Poldi-Pezzoli ligué

(117) Château de Versailles, 10 janvier 1919.

(118) La Lettera fu pubblicata il 14 gennaio 1919 sulla «Gazzetta di Venezia», e il 15 sull'«Idea $\mathrm{Na}$ zionale», sulla «Gazzetta del Popolo» e sul «Popolo d'Italia» diretto da Mussolini.
(119) Château de Versailles, 29 anvier 1919.

(120) [Paris,] 28 décembre 1920

(121) Paris, 6 décembre 1919.

(122) [Paris,] 14 juillet 1922. 
à l'Institut et plein d'oeuvres italiennes)» ${ }^{123}$. In occasione della sua assunzione fra gli immortels scrive a Cian una lettera, quella che servirà come falsariga per l'articolo della «Gazzetta» da cui siamo partiti, che suona quasi come un necrologio:

Je suis ravi de votre bonne pensée! Mais je ne pense pas moi-même sans remords au temps que cela va vous prendre et au nouveau fardeau que votre amical projet ajoute à vos épaules déjà surchargées. Je réponds brièvement (car mes occupations présentes, celles d'un nouvel académicien, ressemblent à celles du directeur du «Giornale Storico»!) aux questions que pose votre amitié. Les Voyages de France et d'Italie sont de 1892. Les Poèmes de F. et d'It. sont de 1905. C'est le même recueil, mais remaniée, diminué et augmenté à la fois.

Erasme en Italie, 1888 - 1898 [...]. Le plus important de mes récents ouvrages sont l'Hist. du Château de Versailles, 3 vol. in $4^{\circ}, 1911-1918$. Les ouvrages de grand luxe sur les peintres [...] sur Boucher, Fragonard, Nattier, Hubert Robert, et $\mathrm{M}^{\mathrm{me}}$ Vigée-Lebrun ${ }^{124}$ (J'ai pris la direction en chef du Musée du Versailles en 1892, l'ai quitté en 1919, aussitôt après la Signature de la Paix, a casa mia).

Savez-vous, carissimo, que Mgr. Duchesne m'avait désigné pour être son successeur à la direction de l'école Française? À l'heure présente encore, le Ministre vaudrait me nommer à ce noble poste, où il y aurait tant de bien à faire pour l'union intellectuelle de nos deux pays. Cette action me tenterait. Mais l'âge est venu, la santé n'est pas solide, j'ai des devoirs de famille (dix petits enfants, ô grand-père Vittorio!). Je crois que je vais me refuser à cet honneur dernier. Mais je compte bien ne pas renoncer à aller en Italie et à Rome (et vous me reverrez à Turin). Je n'ai pas publié mes conférences de la Sapienza, sauf celle sur A. Lorrain (dans les Souvenirs ${ }^{125}$.

Assiste con fervore partecipe, mai sfiorato dal dubbio ( $«$ Je serai bien heureux de voir l'Italie pacifiée, forte et vigoureuse sous ce nouveau régime, qui donne au monde entier le plus magnifique exemple» ${ }^{126}$ ) al trionfo del fascismo: «Je dis volontiers que l'Italie, qui a été si souvent l'école du monde, en est aujourd'hui le modèle... Noi, siamo nel buio ma tornerà il sole» ${ }^{127}$ e i Patti Lateranensi gli dettano un'entusiastica lettera in italiano:

Nei giorni gloriosi che vive adesso l'Italia io penso a te, e indovino la gioia che risente il tuo cuore di credente e di patriota e, anche io, vorrei essere in Roma in questo momento storico per partecipare al sentimento del popolo italiano [...]. Tutto è bene. Grazie siano rendute al Papa e al Re e al Gran Duce d'Italia ${ }^{128}$.

Nella stessa lettera «la bella giornata di Arezzo» diviene «augurio dell'unione che avverrà fra i nostri popoli, malgrado tanti nemici e dificulta [sic]»: bastano a garantirlo «gli avenimenti [sic] presenti [che] son venuti per maturare questo compimento necessario per noi tutti». E quindi con perplesso timore che assiste all'«adhésion du Duce à l'idée germanique de la révision des traités», pur affermando che «on peut croire que l'entente reste possible avec un esprit aussi solide que le sien», nonostante questo atteggiamento sembri «accentuer la divergence de nos politiques» ${ }^{129}$.

La seconda edizione del «petit livre de Souvenirs» gli permette di «parler quotidiennement dans Paris des sentiments qu'il contient et qui se renforcent avec le

(123) Paris, 6 décembre 1919.

(124) P. DE NOLHAC, Boucher, premier peintre du roi, Paris, Floury, 1925; ID., Fragonard (1732-1806), Paris, Goupil, 1918; ID., Nattier: peintre de la cour de Louis XV, Paris, Goupil, 1910; ID., Madame Vigée-Le Brun: peintre de Marie-Antoinette, Paris, Goupil \& C. et Manzi, Joyant \& C., 1908; ID.,
Hubert Robert (1733-1808), Paris, Goupil \& C. et Manzi, Joyant \& C., 1910.

(125) [Paris,] 14 juillet 1922.

(126) Paris, 16 octobre 1923

(127) Paris, 12 janvier 1926.

(128) Paris, 17 février 1929.

(129) [Paris,] 22 décembre 1930. 
temps», e di «constater les erreurs d'une diplomatie française que j'ai toujours combattue». Poco consola il fatto che «on m'assure que M. Briand lui même reconnaît qu'on a fait fausse route envers l'Italie»: la situazione diplomatica resta critica e debolmente gestita: «Il est trop vieux et a trop peu de courage pour revenir sur son passe; mai d'autres le feront» ${ }^{130}$.

In questa ottica assume grande rilevanza il lavoro «bien que beaucoup trop tardif» del secondo comitato Francia-Italia, nato dalla riorganizzazione del primo nel 1929 sotto la direzione di Gabriel Faure ${ }^{131}$ e che «s'impose de plus en plus à une élite d'esprits que je vois grandir autour de nous et qui voit l'inanité de notre politique de sécurité, recherchée en dehors de sa voie naturelle qui est l'amitié italienne». Nolhac si prodiga, temendo in particolare l'azione della stampa che «persuade peu à peu nos peuples de l'imminence d'un conflit armé!»:

Cette monstrueuse pensée n'entre pas dans les esprits chez nous et, si des précautions ont été prises, plus on moins maladroitement, sur nos frontières alpines, ce fut pur rassurer des populations chimériquement inquiétées par le ton de la presse voisine. Je crois sincèrement d'autre part que la fâcheuse alliance yougoslave, faite pour tenir en respect l'Allemagne et ses soutiens balkaniques et nullement contre l'Italie, pourra avoir pour résultat réel de empêcher les Serbes de se livrer à certains écarts et à reffréner [sic] leurs tendances belliqueuses. La diplomatie française ne peut et ne veut servir que la cause de la paix - Je me laisse aller à bavarder avec toi, cher ami, sachant bien que tu ne partageras pas toutes mes idées ${ }^{132}$.

Purtroppo lo stesso Nolhac, in chiusura di questa lettera, è costretto ad ammettere «que bien des apparences semblent me donner tort», «mais - cerca di illudersi - l'avenir latin ne me semble pas compromis» ${ }^{133}$.

Le lettere diventano sempre più rare e spesso solo la firma è autografa: nel 1933 Nolhac ammette: «Ma vue a beaucoup baissé, je ne lis presque plus», ma, si rallegra, «tu vois que je peux encore écrire, tant bien que mal» ${ }^{134}$.

Il «patto a quattro» firmato appena due giorni prima, l'8 giugno del 1933 con la neonata Germania nazista, suscita nuove speranze:

Nous venons de vivre des jours d'émotion heureuse bien longuement, bien péniblement attendus. C'est précisément cette longue maturité de fruit qui permet d'espérer qu'il sera excellent. Tu ne saurais croire quelle impression a produit sur le public français la grandiose manifestation des Sénateurs, pendant le discours du Duce. Cette marque si éloquente d'amitié désirée a touché bien des cœurs hésitants, que le préjugé antifasciste obscurcit encore. Il m'était particulièrement doux de penser aux amis qui se levaient sur les bancs du Sénat, autour de l'admirable chef, et de savoir que tu étais du nombre, mon cher Victor. Nous ne devons pas ignorer qu'il y aura encore à combattre pour la bonne cause; mais, dès à présent, le terrain est déblayé pour le travail commun, et Mussolini, qui a pris cette grande initiative, apparaît comme l'incarnation la plus haute du génie latin ${ }^{135}$.

L'accordo con il ministro degli esteri francese Pierre Laval, che legittima un rafforzamento italiano in Etiopia, gli fa esclamare, a lui che «espérait vivre assez

(130) Ibidem

(131) Il primo comitato, nato sotto la presidenza del senatore Stéphen Pichon, aveva un suo corrispondente in Italia di cui facevano parte Visconti Venosta, G. Ferrero, F. De Marinis, P. Molmenti e A. Graf. Per le vicende legate alle riviste vicine a queste iniziative, il «Bulletin italien» prima e la «Revue des études italiennes» poi, cfr. C. DEJOB, Histoire de la So- cieté d'Etudes Italiennes, Paris, E. de Boccard, 1919 e R. De CESARE, Il «Bulletin italien» (1901-1918), in Critica e storia letteraria. Studi offerti a Mario Fubini, II, Padova, Liviana, 1970, pp. 69-111.

(132) [Paris,] 22 décembre 1930.

(133) Ibidem.

(134) Paris, 10 juin 1933.

(135) Paris, 10 juin 1933. 
pour assister au revirement nécessaire ${ }^{136}$, «Nunc dimittis servum tuum, Domine» ${ }^{137}$, quasi la sua missione personale e quella del comitato fossero esaurite. Ma ben presto si deve ricredere: «J'attends impatiemment que la foudre mussolinienne tombe sur la mitre du lion barbare ${ }^{138}$ scrive nel luglio dello stesso anno, il 1935 , quando comprende l'inutilità degli accordi di Stresa. La storia, suo malgrado, sta andando in un'altra direzione e, quando nel 1936 Nolhac scompare, non basta certo il messaggio di Mussolini alla vedova ad attenuare «le silence glacial qui pesait entre les deux pays» ${ }^{139}$.

Si tratta di un «silence» che, sia pure con una forma di engagement tutta particolare, Nolhac aveva cercato di vincere. Non siamo certo di fronte a un livello di consapevolezza simile a quello di Romain Rolland, che il 26 giugno 1919 aveva pubblicato sull'«Humanité» la Déclaration d'indépendance de l'esprit, recentemente riproposta da Lionello Sozzi ${ }^{140}$ per la sua impressionante attualità. D'altronde alcune delle argomentazioni utilizzate da Rolland, che sarebbero potute egregiamente servire ai fautori di un riavvicinamento italo-francese sulla fine degli anni Trenta, non erano state comprese appieno fin dai tempi di Au-dessus de la mêlée. Infatti nel 1918 Cian, notoriamente nazionalista e interventista, accusava Croce dalle pagine del «Giornale storico» di atteggiamenti «alla Romain Rolland, punto simpatici e punto opportuni in tempi come questi, nei quali il voler porsi al disopra o al difuori della lotta è un porsi al disotto e al difuori dell'umanità e della Patria» ${ }^{141}$, e, in una lettera privata, sempre a Croce, ribadiva che

la questione è [...] della convenienza e della utilità di certi atteggiamenti al di sopra della lotta, alla Romain Rolland, in tempi nei quali tutto quanto è di meglio nelle energie della nazione in lotta per la sua esistenza - tutto, ingegno, volontà, scienza e arte, individui e collettività - deve servire alla vittoria ${ }^{142}$.

Croce, dal canto suo, aveva reagito duramente ricordando di aver «sempre combattuto» Rolland «nelle postille della Critica» in quanto «dava torto a tutti i popoli combattenti, e lui ragione a tutti, perché tutti difendevano le loro patrie e combattevano per la grandezza di esse» ${ }^{143}$. Anche se il suo nome sarebbe poi comparso nel 1919 in calce alla Déclaration, il filosofo napoletano non sembrava cogliere quegli aspetti che, nella critica dell'intellettuale francese, trascendevano il contesto del primo conflitto mondiale.

E quando il 10 giugno 1940 l'Italia dichiarò guerra alla Francia, il disorientamento, spesso taciuto, di molti «travailleurs de l'Esprit» a cui la Déclaration era indirizzata, si fece manifesto di fronte alla caduta di un tabù che si pensava inviolabile. Lo stesso Nolhac, se fosse sopravvissuto fino ad allora, avrebbe forse potuto comprendere quanto fragile e illusorio fosse stato negli ultimi anni il suo rapporto con il potere,

(136) [Paris,] 22 décembre 1930.

(137) Cartolina indirizzata «all'amico carissimo per sempre Vittorio Cian», in data 7 gennaio 1935 , giorno dell'accordo, custodita alla Fondazione Cini.

(138) Paris, 15 juin 1935

(139) G.E. BROCHE, Quelques lettres de Pierre de Nolbac, cit., p. 56.

(140) L. SozzI, Romain Rolland e l'«indipendenza dello spirito», in La letteratura dell'«impegno» nel Novecento europeo, a cura di M. SPADARO, pref. di L. Sozzi, Alessandria, Edizioni dell'Orso, 2007.

(141) V. Cian, In cammino..., «Giornale storico della letteratura italiana», 72 (1918), pp. 225-244.

(142) B. Croce-V. Cian, Carteggio, cit., lettera ${ }^{\circ}$ 325.

(143) Ivi, lettera $n^{\circ} 333$. In una lettera a Romain Rolland del 9 aprile del 1919, trascritta alla p. 31 nell'intervento di Sozzi citato alla nota 140, Croce, in un post scriptum, ironizzava proprio su questo avvicinamento: «a proposito di "fraternità", se le può far piacere la informo che, durante la guerra, nei più accesi giornali italici, tornava frequente la frase: "Quei due perfetti idioti (pardon!) che rispondono al nome di Romain Rolland e Benedetto Croce". Si può essere affratellati in modo più compiuto di così?». 
rapporto imprescindibile, è ancora Sozzi a ricordarcelo ${ }^{144}$, da ogni impegno. Quattro anni prima di morire, nel 1932, raccogliendo in volume i suoi Contes philosophiques per i tipi di Bernard Grasset, Nolhac scriveva a Gaston Broche d'«hésiter à répandre en Italie, [...] bien qu'il y ait près de la moitié du volume intéressant le cher pays» e il destinatario chiosava «en souriant»: «Habemus confitentem reum!».

Eppure, poche righe prima lo stesso Broche aveva citato L'écolier d'Avignon, definendolo «le chant du cygne du grand pétrarquisant», senza comprendere che «le jeu trop hardi» ${ }^{145}$ che aveva ispirato la Nuit era esattamente lo stesso che portava il passato e il presente a incontrarsi in una dimensione in cui fossero salvi tutti gli elementi cari al poeta e all'uomo. Qualcosa di simile accade nel Testament d'un latin, dove, dice André Pératé, Nolhac «a résumé ce qui fut l'enseignement, reçu et donné, de sa vie, hommage à l'esprit vivant qui, aux rives de la Méditerranée, depuis la Grèce antique jusqu'à nous, a germé, a fleuri» ${ }^{146}$.

Non c'è allora molta differenza fra Nolhac, privato del «son maître» dall'«appel de son pays» e il Dr. Colombe che avevamo lasciato a «décrocher son trousseau de clés», per condurre il papa attraverso le «vastes salles désertes», «indiquant en chemin les degrés et les passages qui pourraient le conduire [...] à la Tour des Anges, à la Bibliothèque, à la Chapelle Saint-Jean». Dopo questa breve parentesi l'erudito scompare dalla Nuit: ha solo «fatto gli onori di casa» ${ }^{147}$. Ma ha avuto il tempo di confessarci il suo «songe» più riposto e impossibile: «recevoir la visite du Pape» ${ }^{148}$, così come l'«écolier d'Avignon» sognava di «rencontrer dans la maison du Pape Maître François» ${ }^{149}$.

CLARA ALLASIA

(144) L. Sozzi, Presentazione, in La letteratura dell'«impegno» nel Novecento europeo, cit., p. VII.

(145) G.E. BROCHE, Quelques lettres de Pierre de Nolbac, cit., p. 53.

(146) A., PÉRATÉ, Pierre de Nolhac, un grand bumaniste, «Études», 20 mars 1936, p. 791.

(147) V. Cian, Pierre de Nolhac, cit.

(148) P. De Nolhac, La nuit de Pie XII, cit., p. 485.

(149) ID., L'écolier d'Avignon (Vaucluse 1352), cit., p. 173.

Desidero ringraziare il professor Angelo Raffaele Meo, presidente dell'Accademia delle Scienze di Torino e il professor Pietro Rossi, suo predecessore, che mi hanno permesso di utilizzare il materiale inedito qui trascritto. Particolare gratitudine devo al dottor Franco Casini della Biblioteca di Lettere e teatro della Fondazione Cini di Venezia, alla dottoressa Rosangela Risso e a tutto il personale della Biblioteca della Facoltà di Lettere e Filosofia dell'Università di Torino, alla dottoressa Elena Borgi responsabile della Biblioteca dell'Accademia delle Scienze, alla dottoressa Chiara Caprioglio e al dottor Pier Mario Arscone della Biblioteca "Gioele Solari" dell'Università di Torino, che mi hanno aiutato nel difficile reperimento di alcune opere utili a questo lavoro. L'amica Maria Cristina Icardi mi ha assistito nella decifrazione della non sempre leggibile grafia di Nolhac. Alla professoressa Laura Nay un pensiero sinceramente grato per il prezioso aiuto e i concreti suggerimenti. Al professor Lionello Sozzi, che ha voluto incoraggiarmi a procedere in questa ricerca "francese", la mia più sentita riconoscenza. 